\title{
Lipid-lowering pharmacotherapy and socioeconomic status: atherosclerosis risk in communities (ARIC) surveillance study
}

\author{
Joseph P Kitzmiller ${ }^{1}$, Randi E Foraker ${ }^{2}$ and Kathy M Rose $34^{3 *}$
}

\begin{abstract}
Background: Lipid-reduction pharmacotherapy is often employed to reduce morbidity and mortality risk for patients with dyslipidemia or established cardiovascular disease. Associations between socioeconomic factors and the prescribing and use of lipid-lowering agents have been reported in several developed countries.

Methods: We evaluated the association of census tract-level neighborhood household income (nINC) and lipidlowering medications received during hospitalization or at discharge among 3,546 (5,335 weighted) myocardial infarction (MI) events in the United States (US) Atherosclerosis Risk In Communities (ARIC) surveillance study (1999-2002). Models included neighborhood household income, race, gender, age, study community, year of Ml, hospital type (teaching vs. nonteaching), current or past history of hypertension, diabetes or heart failure, and presence of cardiac pain.

Results: About fifty-nine percent of patients received lipid-lowering pharmacotherapy during hospitalization or at discharge. Low nINC was associated with a lower likelihood (prevalence ratio 0.89, 95\% confidence interval: 0.79, 1.01) of receiving lipid-lowering pharmacotherapy compared to high neighborhood household income, and no significant change in this association resulted when adjusted for the above-mentioned covariates.
\end{abstract}

Conclusion: Patient's socioeconomic status appeared to influence whether they were prescribed a lipid-lowering pharmacotherapy after hospitalization for myocardial infarction in the US ARIC surveillance study (1999-2002).

Keywords: Lipids, Socioeconomics, Statins, Lipid-lowering pharmacotherapy, Cardiovascular disease

\section{Background}

Lipid reduction can significantly improve cardiovascular risk and lower morbidity and mortality following myocardial infarction (MI). Pharmacotherapy, along with lifestyle changes, plays an essential role in improving lipid profiles. Statins, arguably the most efficacious of the lipid-lowering drug classes, are often first-line therapy for the dyslipidemias and for decreasing cardiovascular risk. However, investigators worldwide have reported that socioeconomic factors often influence the prescribing and use of statins and other lipid-lowering agents. In 2007 Ward et. al. published data from four primary-care trusts in Northwest England that indicated

\footnotetext{
*Correspondence: kathy_rose@sra.com

${ }^{3}$ Department of Epidemiology, Gillings School of Global Public Health, University of North Carolina, 135 Dauer Drive, Chapel Hill, NC 27599, USA ${ }^{4}$ SRA International, Inc, 2605 Meridian Parkway, Durham, NC 27713, USA Full list of author information is available at the end of the article
}

ethnic inequities in statin prescribing rates [1]. A Danish study published in 2005 reported that among men with cardiovascular disease, statin use was higher in those with the highest socioeconomic status (SES) and lower among retired men in old-age pensioners compared to basic-level workers [2]. An Australian study published in 2004 found that statins were prescribed for males when indicated more often to those with higher SES and were prescribed for females at higher rates at lower levels of risk [3]. In 2006 a report describing significant disparities in the use of lipid-lowering agents in the United States (US) was published [4]; however, such studies investigating SES and the use of lipid-lowering medications in the US are rare. Our previous work described differential receipt of aspirin, beta-blockers, and angiotensin converting enzyme inhibitors by neighborhood SES [5]. This brief report discusses our subsequent

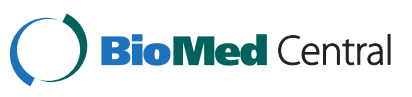


investigation into the relationship between neighborhood SES and lipid-lowering pharmacotherapy.

\section{Methods}

We evaluated the association between tertiles (low, medium, and high) of census tract-level neighborhood household income (nINC) and lipid-lowering medications received during hospitalization or at discharge among 3,546 (5,335 weighted) MI events in the US Atherosclerosis Risk In Communities (ARIC) surveillance study (1999-2002). The ARIC study's community-based surveillance of coronary heart disease has been ongoing since 1987 and is designed to capture MI and fatal coronary heart disease events in four US communities [Jackson,

Table 1 Receipt of lipid-lowering pharmacotherapy among Atherosclerosis Risk in Community (ARIC) surveillance patients (1999-2002): results from regression models for selected variables

\begin{tabular}{|c|c|c|}
\hline Variable & Prevalence ratio & $95 \% \mathrm{Cl}$ \\
\hline \multicolumn{3}{|l|}{ Model 1} \\
\hline \multicolumn{3}{|l|}{$\mathrm{nINC}$} \\
\hline Low nINC & 0.89 & $0.79,1.01$ \\
\hline Medium nINC & 0.98 & $0.91,1.07$ \\
\hline High nINC & 1.00 & ref \\
\hline \multicolumn{3}{|l|}{ Gender } \\
\hline Female & 0.91 & $0.84,0.98$ \\
\hline Male & 1.00 & ref \\
\hline \multicolumn{3}{|l|}{ Race } \\
\hline Black & 0.86 & $0.76,0.97$ \\
\hline White & 1.00 & ref \\
\hline \multicolumn{3}{|l|}{ Model 2} \\
\hline \multicolumn{3}{|l|}{$\mathrm{nINC}$} \\
\hline Low nINC & 0.91 & $0.81,1.03$ \\
\hline Medium nINC & 1.00 & $0.92,1.08$ \\
\hline High nINC & 1.00 & ref \\
\hline \multicolumn{3}{|l|}{ Gender } \\
\hline Female & 0.92 & $0.85,1.00$ \\
\hline Male & 1.00 & ref \\
\hline \multicolumn{3}{|l|}{ Race } \\
\hline Black & 0.90 & $0.79,1.02$ \\
\hline White & 1.00 & ref \\
\hline \multicolumn{3}{|l|}{ Hospital type } \\
\hline Teaching & 0.87 & $0.79,0.96$ \\
\hline Non-teaching & 1.00 & ref \\
\hline \multicolumn{3}{|l|}{ Medical history } \\
\hline Hypertension & 0.99 & $0.92,1.06$ \\
\hline Diabetes & 1.05 & $0.97,1.14$ \\
\hline Heart failure & 0.72 & $0.65,0.79$ \\
\hline
\end{tabular}

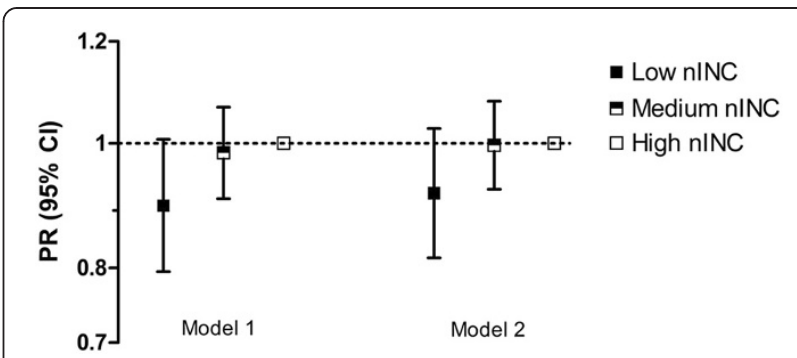

Figure 1 Receipt of lipid-lowering pharmacotherapy among Atherosclerosis Risk in Community (ARIC) surveillance patients (1999-2002). Model 1: Neighborhood income (nINC), race, gender, age, study community, year of myocardial infarction (MI). Model 2: Model 1 plus hospital type (teaching vs. nonteaching), current or past history of hypertension, diabetes or heart failure, and presence of cardiac pain.

Mississippi (MS); Forsyth County, North Carolina (NC); Washington County, Maryland (MD); and Minneapolis, Minnesota (MN)]). While it comprises the same communities from which ARIC cohort members were recruited, ARIC community surveillance does not include in-person physical exams, annual follow-up, or any contact with ARIC cohort participants (unless they happen to be sampled as a surveillance case). ARIC community-surveillance staff ascertained coronary heart disease-related hospital discharges and deaths and abstracted data related to the event of interest. Institutional Review Board (IRB) approvals were obtained by each participating ARIC study center (the Universities of NC, MS, MN, and John Hopkins University) and the coordinating center (University of NC), and the research was conducted in accordance with the principles described in the Declaration of Helsinki. Data for this study were abstracted from medical records and strict data confidentiality was maintained. Further details regarding ARIC's methods for data collection are provided elsewhere [6]. For our analyses, we weighted the hospitalized MI cases based on the probability sampling of selected International Classification of Disease codes [6] in order to estimate the eligible population of cases that would have been studied had the probability sampling not been employed.

We estimated prevalence ratios and 95\% confidence intervals for receipt of lipid-lowering medication postMI using weighted Poisson regression, and we used generalized estimation equations (PROC GENMOD, SAS Institute) to account for the clustering of MI events within census tracts and within patients, as incident and recurrent MI events were considered together based upon our previous analyses $[7,8]$. Model 1 included nINC, race, gender, age, study community and year of MI. Model 2 included the same parameters as well as hospital type (teaching vs. nonteaching), current or past history of hypertension, diabetes or heart failure, and presence of cardiac pain. Additionally, we examined 
whether the following parameters modified the nINC lipid-lowering therapy relationship: race, gender, age, study community and year of MI. Models utilizing tertiles defined by overall nINC cut-points were evaluated, as interpretations of our earlier work in this population did not change based on the delineation (community-specific, race-specific, and overall cut-points) of nINC tertiles [9].

\section{Results and discussion}

The MI cases were distributed across the four ARIC study communities as follows: $25.7 \%$ in MS, $36.7 \%$ in NC, $15.9 \%$ in MD, and $21.7 \%$ in MN. The MI patients were $34.5 \%$ female, $25.3 \%$ black, and had an average age of 60.2 years. The mean nINC was approximately $\$ 42,700$. Overall, 58.5\% of patients received lipid-lowering pharmacotherapy during hospitalization or at discharge. In models adjusted for race, gender, age, study community and year of MI, there was no significant effect modification of the nINC- lipid-lowering therapy relationship by race, gender, age, study community or year of MI as indicated in these data. Results of models utilizing overall cutpoints are reported in Table 1 and Figure 1. In Model 1, low nINC was associated with a lower likelihood (prevalence ratio $0.89,95 \%$ confidence interval: $0.79,1.01$ ) of receiving lipid-lowering pharmacotherapy compared to high nINC. This relationship did not change substantially when the additional parameters (hospital type, current or past history of hypertension, diabetes or heart failure, and presence of cardiac pain) were included (Model 2). Although lipid-lowering pharmacotherapy remains a central component of cardiovascular-risk-profile optimization, several investigators worldwide have reported significant associations among socioeconomic parameters and statin prescribing. Our investigation suggests that prescribing of lipid-lowering pharmacotherapies may be influenced by neighborhood SES in the US ARIC study population (1999-2002).

\section{Conclusions}

Our results demonstrate that a patient's SES may influence whether they were prescribed a lipid-lowering pharmacotherapy after hospitalization for MI in the US ARIC surveillance study (1999-2002). This observation should be noted by clinicians, patients, and policy makers. Efforts to decrease or eliminate the influence of socioeconomic factors on the prescribing of pharmacotherapy should be implemented.

\section{Abbreviations}

US: United States; ARIC: Atherosclerosis risk in communities; MI: Myocardial infarction; SES: Socioeconomic status; nINC: neighborhood household income; MS: Mississippi; NC: North Carolina; MD: Maryland; MN: Minneapolis.

\section{Authors' contributions}

JPK drafted the manuscript and contributed to the analysis and interpretation of data. REF participated in the design of the study, acquired the data, performed the data analysis and interpretation, and assisted in drafting and revising the manuscript. KMR participated in the design of the study, assisted in the data analysis and interpretation, and also assisted in revising the manuscript. All three authors certify that they have participated sufficiently in the work and believe in its overall validity and take public responsibility for its content. All authors read and approved the final manuscript.

\section{Acknowledgements}

The authors thank the staff of the ARIC study for their important contributions. This research was supported by NHLBI grant 1R01HL080287 and by NIGMS grant K23GM100372. It was also funded in part by National Institutes of Health, NHLBI, and National Research Service Award training grant 5-T32-HL007055-30.

The Atherosclerosis Risk in Communities Study is carried out as a collaborative study supported by National Heart, Lung, and Blood Institute contracts N01-HC-55015, N01-HC-55016, N01-HC-55018, N01-HC-55019, N01HC-55020, N01-HC-55021, and N01-HC-55022.

\section{Author details}

'Departments of Pharmacology and Biomedical Engineering, The Ohio State University Medical Center, 5072B Graves Hall 333 West 10th Avenue, Columbus, $\mathrm{OH} 43210$, USA. ${ }^{2}$ Division of Epidemiology, College of Public Health, The Ohio State University, 334 Cunz Hall, 1841 Neil Avenue, Columbus, $\mathrm{OH} 43230$, USA. ${ }^{3}$ Department of Epidemiology, Gillings School of Global Public Health, University of North Carolina, 135 Dauer Drive, Chapel Hill, NC 27599, USA. ${ }^{4}$ SRA International, Inc, 2605 Meridian Parkway, Durham, NC 27713, USA.

Received: 2 December 2012 Accepted: 16 May 2013

Published: 20 May 2013

\section{References}

1. Ward PR, Noyce PR, Leger AS: How equitable are GP practice prescribing rates for statins?: an ecological study in four primary care trusts in north west England. Int J Equity Health 2007, 6:2

2. Thomson RW, Johnsen SP, Olesen AV, Mortensen JT, Boggild H, Olsen J, Sorensen HT: Socioeconomic gradient in use of statins among Danish patients: population-based cross-sectional study. Br J Clin Pharmacol 2005, 60:534-542.

3. Stocks N, Ryan P, McElroy H, Allan J: Statin prescribing in Australia: socioeconomic and sex differences. Med J Australia 2004, 180:229-231.

4. Litaker D, Koroukian S, Frolkis JP, Aron DC: Disparities among the disadvantaged: Variation in lipid management in the Ohio Medicaid Program. Preventative Med 2006, 42:313-315.

5. Foraker RE, Rose KM, Whitsel EA, Suchindran CM, Wood JL, Rosamond WD: Neighborhood socioeconomic status, Medicaid coverage and medical management of myocardial infarction: atherosclerosis risk in communities (ARIC) community surveillance. BMC Public Health 2010 10:632.

6. The ARIC investigators: The Atherosclerosis Risk in Communities (ARIC) Study: design and objectives. Am J Epidemiol 1989, 4:687-702.

7. Bryk A, Raudenbush A: Hierarchical linear models: applications and data analysis methods. Newbury Park, CA: Sage Publications; 1992.

8. Williams RL: A note on robust variance estimation for cluster-correlated data. Biometrics 2000, 56:645-646.

9. Foraker RE, Rose KM, Kucharsska-Newton AM, Ni H, Suchindran CM, Whitsel EA: Variation in rates of fatal coronary heart disease by neighborhood socioeconomic status: the atherosclerosis risk in communities surveillance (1992-2002). Ann Epidemiol 2011, 21(8):580-588.

doi:10.1186/1471-2458-13-488

Cite this article as: Kitzmiller et al:: Lipid-lowering pharmacotherapy and socioeconomic status: atherosclerosis risk in communities (ARIC) surveillance study. BMC Public Health 2013 13:488. 